\section{FRI0152 RHEUMATOLOGISTS' EXPERIENCES AND VIEWPOINTS TOWARDS MANAGING RHEUMATOID ARTHRITIS IN ELDERLY PATIENTS: A QUALITATIVE STUDY}

J. Nawrot ${ }^{1}$, A. Boonen ${ }^{1,2}$, R. Peeters ${ }^{3}$, M. Starmans ${ }^{3}$, M. Van Onna ${ }^{1,2}$ ${ }^{1}$ Department of Internal Medicine, division of Rheumatology, Maastricht University Medical Center $+;^{2}$ School for Public Health and Primary Care, Maastricht University, Maastricht; ${ }^{3}$ Rheumatology, Zuyderland Hospital, Heerlen, Netherlands

Background: The number of elderly individuals with rheumatoid arthritis (RA) is expanding in Europe, mainly due to an increase in life expectancy. As a result, management of these patients, e.g. the application of the treat-to-target and tight control principles, shall have to account for frailty and comorbidity. However, knowledge about how rheumatologists perceive and manage RA in the elderly patient population is limited.

Objectives: To explore the viewpoints of rheumatologists on management goals in elderly RA patients and the influence of factors such as age, frailty and comorbidity on these goals. Furthermore, experiences of rheumatologists with regard to outcome instruments to guide management in elderly patients were assessed. Methods: A qualitative study involving semi-structured interviews with rheumatologists was conducted. Two readers independently annotated the transcripts of the interviews. Important concepts were taxonomically categorized and later combined in overarching themes by using NVivo 11.

Results: Seventeen rheumatologists were purposively sampled from nine medical centres (mean age: 44.8 years (SD 7.7 years); $29 \%$ male). High levels of frailty and comorbidity frequently influenced management goals of rheumatologists: in these cases, preserving an acceptable functional status prevailed over the treat-to-target and tight control principles. For instance, most rheumatologists accepted the presence of tender and swollen joints when overall functioning and social participation were not or only minimally impaired. In patients $>80$ years, age instead of frailty and comorbidity was the most prominent factor that steered management. On that line, almost all rheumatologists admitted that their management strategy is less driven by the result of the Disease Activity Score-28 (DAS28), since comorbidity (e.g. osteoarthritis) and an age-related physiological Erythrocyte Sedimentation Rate (ESR)-elevation might distort the DAS28 value. Instead, before adapting anti-rheumatic therapy, rheumatologists weighted the frailty and comorbidity levels of a patient and the functional consequences of these factors such as cognitive and physical decline, dependency and polypharmacy (quote 1, Table 1). This frequently resulted in a less future-oriented management approach that was not aimed at the maximal prevention of joint erosions and deformities (quote 2, Table 1). Rheumatologists reported that a lack of time to evaluate all comorbid conditions, as well as contradictory advices of other medical specialists often complicated the management of elderly RA patients.

Table 1. Illustrative quotes made by rheumatologists

\begin{tabular}{|l|l|}
\hline Quote & \\
\hline 1 & $\begin{array}{l}\text { I sometimes ask my patients: what physical complaint bothers you the most? The } \\
\text { RA, the heart failure or something else? Treat what actually limits an elderly } \\
\text { patient.' }\end{array}$ \\
\hline 2 & $\begin{array}{l}\text { 'I wonder if low-dosage prednisone for the rest of their lives is considerably worse } \\
\text { than to initiate biological therapy and cause a life-threatening infection?' }\end{array}$ \\
\hline
\end{tabular}

Conclusions: Commonly accepted RA treatment paradigms such as treat-totarget and tight control are not automatically adopted in the elderly patient population. Maintaining a patient acceptable functional status prevails. Future RA management recommendations for elderly RA patients are needed and should account for factors such as frailty and comorbidity.

Disclosure of Interest: None declared

DOI: 10.1136/annrheumdis-2017-eular.2291

\section{FRI0153 COMPARISON OF CARDIOVASCULAR RISK IN PATIENTS WITH RHEUMATOID ARTHRITIS TREATED WITH BIOLOGICS VERSUS METHOTREXATE: RESULTS AT 24-MONTHS OF FOLLOW-UP}

M. Khraishi ${ }^{1}$, M. Stutz ${ }^{2}$, A. Lewis ${ }^{3}$, C. Molta ${ }^{4}$, E. Rampakakis ${ }^{2} .{ }^{1}$ Rheumatology, Memorial University of Newfounbdland, St. John's; ${ }^{2}$ JSS Medical Research, St-Laurent; ${ }^{3}$ Epidemiology, Memorial University of Newfoundland, St.John's, Canada; ${ }^{4}$ Rheumatology, MainLine Rheumatology Lankenau Medical Center, Philadelphia, United States

Background: Rheumatoid arthritis (RA) is associated with increased risk of atherosclerotic cardiovascular (CV) disease. Treatment with conventional systemic disease modifying drugs (csDMARDs) such as methotrexate (MTX), as well as biological DMARDs (bDMARDs), has been shown to decrease CV risk. Although bDMARDs specifically target inflammation common to RA and atherosclerosis, whether or not cardioprotective effects associated with bDMARD use is superior to csDMARDs, remains to be determined.

Objectives: To investigate 10 year-CV risk, and incidence of new myocardial infarctions (MI), in RA patients treated with either MTX or bDMARD mono/combination therapy, in a Canadian routine clinical care setting

Methods: RA patients were prospectively followed between January 2011-March
2014. Parameters collected were patient demographics, RA disease activity parameters, traditional CV risk factors, lipid parameters, and 10-year CV risk, assessed using the Framingham Risk Score (FRS). Between-group differences in change from baseline to month 24 in FRS, disease activity, and lipid parameters were assessed with the two sample t-test or the Chi-Square statistic; within-group differences were assessed with the paired-samples t-test or the McNemar-Bowker test. Adjusted change in FRS was ascertained using general linear models, and logistic regression identified predictors of MI.

Results: A total of 517 RA patients receiving bDMARDs $(n=313)$ or MTX $(n=199)$, were included. Mean (SD) age was comparable between cohorts [57.57 (12.11) years vs. 59.58 (12.60), $p=0.11$; bDMARD vs. MTX, respectively], as was female gender $(76.7 \%$ vs. $73.9 \%, p=0.268)$. Patients receiving bDMARDs had significantly $(p<0.05)$ longer mean (SD) RA duration [12.63 (9.95) vs. 7.88 (6.94) years] and higher total comorbidities [3.96 (2.53) vs. 3.38 (2.32)]. Mean (SD) baseline FRS was 11.84 (9.38) vs. 12.36 (9.19) percent ( $p=0.564$; bDMARDs vs. MTX, respectively), and patient distribution across low (62.3\% vs. $54.8 \%)$, intermediate (9.9\% vs. $12.2 \%)$ and high $(27.8 \%$ vs. $33.2 \%)$ FRS risk categories was comparable $(\mathrm{p}=0.239)$.

At month 24, FRS category remained stable in bDMARD patients (low: $58.8 \%$; intermediate: $14.2 \%$; high: $27.0 \% ; \mathrm{p}=0.380$ ), whereas a shift in FRS category was observed in MTX patients (low: 69.6\%; intermediate: $10.1 \%$; high: $27.0 \%$, $\mathrm{p}=0.006)$. Within-group changes in FRS were significant for both MTX $(p<0.001)$ and bDMARD patients $(p=0.016)$. Adjusted mean change (SE) in FRS was higher in MTX patients [-1.37 (0.30) vs. $-0.72(0.25)]$, although not statistically different $(p=0.098)$. Incidence of new Ml was similar between groups (MTX: $3.8 \%$ vs. dDMARD: $3.0 \%$; $=0.421)$, and predictors [OR $(95 \% \mathrm{Cl})$ ] identified were: higher total comorbidities [1.45 $(1.24,2.20), p<0.001]$, age [1.07 $(1.00,1.13), p=0.037]$, and male gender $[4.00(1.38,11.57), p=0.011]$.

Conclusions: Significant improvement in CV risk at 24 months was observed during treatment with both MTX and bDMARDs. As predictors of MI did not include several established CV risk factors, longer studies, as well as the development of an RA-specific tool, may permit better assessment of CV risk in RA patients.

Disclosure of Interest: M. Khraishi Grant/research support from: Roche Canada, M. Stutz: None declared, A. Lewis: None declared, C. Molta: None declared, E. Rampakakis: None declared

DOI: 10.1136/annrheumdis-2017-eular.2789

\section{FRI0154 THE TOLERABILITY OF VACCINATION AGAINST PNEUMOCOCCUS IN CHILDREN WITH JUVENILE IDIOPATHIC ARTHRITIS}

M. Soloshenko ${ }^{1}$, E. Alexeeva ${ }^{1,2}$, T. Bzarova ${ }^{1,2}$, S. Valieva ${ }^{1}$, R. Denisova ${ }^{1}$, O. Lomakina ${ }^{1}$, K. Isaeva ${ }^{1}$, E. Kashchenko ${ }^{1}$, A. Karaseva ${ }^{1}$. ${ }^{1}$ Federal State Autonomous Institution "National Scientific and Practical Center of Children's Health"; ${ }^{2}$ I.M. Sechenov First Moscow State Medical University, Moscow, Russian Federation

Background: Children with chronic rheumatic diseases, including juvenile idiopathic artritis, are at high risk of bacterial and viral infections. The risk of complications and severity of infectious diseases are increased in immunosuppressive therapy due to given the long-term use.

Objectives: To evaluate the safety and tolerability of vaccination against pneumococcus in children with juvenile idiopathic arthritis.

Methods: The analysis included 39 patients with juvenile idiopathic arthritis who were in remission. Patients were divided into two groups, depending on the type of immunosuppressive therapy. Group $1(n=24)$ consisted of patients receiving etanercept, mean age $5,4 \pm 2,5$, observed 48 patient-years; Group 2 $(n=15)$, patients who received methotrexate mean age $6,8 \pm 3,2$, observed 30 patient-years. All patients had been vaccinated by 13 -valent pneumococcal polysaccharide conjugate vaccine (13PPV). Recorded the incidence of infection diseases (such as pneumonia, tonsillitis, otitis media, etc.) in the 12 months prior to vaccination and 12 months after vaccination. All patients monitored the development of adverse events against the background of vaccination.

Results: The study showed a decrease in the incidence ofinfection diseases after vaccination in the first group from $5,4 \pm 1,66$ to $1,7 \pm 0,77$, in second group from $4,4 \pm 1,05$ to $1,6 \pm 073,(p<0.05)$. Adverse events related to vaccination are presented in the table. Results of the study showed no relapse of the underlying disease following immunization. Adverse events related to vaccination are presented in the table.

Conclusions: The results showed a good tolerability of the vaccine, no relapse of the underlying disease following immunization, as well as a statistically significant $(p<0.05)$ reduction in the incidence following immunization.

Disclosure of Interest: M. Soloshenko: None declared, E. Alexeeva Grant/research support from: Roche, Abbott, Pfizer, Bristol-Myers Squibb, Centocor, Novartis, Speakers bureau: Roche, Merck Sharp \& Dohme, Abbott, Bristol-Myers Squibb, Medac, Novartis, Pfizer, T. Bzarova Grant/research support

\begin{tabular}{lcc}
\hline Adverse events & Group I: Methotrexate & Group II: Etanercept \\
\hline Total, $\mathrm{n}$ & 6 & 3 \\
Hyperemia and soreness at the injection site & 3 & 2 \\
Swelling at the site of vaccine injection & 1 & 1 \\
Fever & 1 & - \\
Headache & 1 & - \\
\hline
\end{tabular}

